\title{
High Body Mass Index in Elderly Patients With DLBCL Treated With Rituximab- Containing Therapy Compensates for Negative Impact of Male Sex
}

\author{
Zheng Zhou, MD, PhD; ; Alfred W. Rademaker, PhD; ${ }^{\mathrm{a}}$, Leo I. Gordon, MDa; Ann S. LaCasce, MD; \\ Allison Crosby-Thompson, MSc ${ }^{\mathrm{b}}$; Ann Vanderplas, $\mathrm{MSc}^{\mathrm{c}}$; Gregory A. Abel, MD ${ }^{\mathrm{b}}$; Maria A. Rodriguez, MD \\ Auayporn Nademanee, MD; ${ }^{\mathrm{e}}$ Mark S. Kaminski, MD; Myron S. Czuczman, MD;; \\ Michael M. Millenson, $\mathrm{MD}^{\mathrm{h}}$; Andrew D. Zelenetz, MD, PhDi; Joyce Niland, $\mathrm{PhD}^{\mathrm{c}}$; \\ Jonathan W. Friedberg, MD, MMSc'; and Jane N. Winter, $\mathrm{MD}^{\mathrm{a}}$
}

\begin{abstract}
Background: The impact of patient body habitus and sex on outcomes in diffuse large B-cell lymphoma (DLBCL) remains controversial. We investigated the impact of body mass index (BMI), body surface area (BSA), age, and sex on clinical outcomes in patients with DLBCL treated in the rituximab era. Patients and Methods: Patients with de novo DLBCL $(n=1,386)$ diagnosed between June 2000 and December 2010 treated with rituximab-containing chemotherapy were identified from the NCCN Oncology Outcomes Database for Non-Hodgkin's Lymphoma. Progression-free survival (PFS) and overall survival (OS) at 3 years were analyzed based on sex, age, and baseline BMI/BSA. Results: High BMI was associated with a lower risk of disease progression or death than low or normal BMI, whereas male sex was associated with poor clinical outcomes, especially among elderly patients (age $>60$ years). Compared with elderly women, elderly men experienced worse PFS (3-year hazard ratio [HR], 1.5) and OS (3-year HR, 1.6), but these differences diminished with increases in BMI and BSA. In multivariable analysis, normal BMI compared with high BMI was independently associated with poor outcomes (3-year PFS HR, 1.5; OS HR, 1.6) after adjusting for sex. Notably, only $13 \%$ of elderly men had BMI less than $25 \mathrm{~kg} / \mathrm{m}^{2}$ and only $26 \%$ had BSA less than $2 \mathrm{~m}^{2}$. Conclusions: Analysis of unselected patients with DLBCL treated with rituximab-containing chemotherapy confirmed an age-dependent disadvantage to male sex in treatment outcomes, but this effect is abrogated by higher levels of BMI and BSA in most North American men.
\end{abstract}

J Natl Compr Canc Netw 2016;14(10):1274-1281

From aRobert H. Lurie Comprehensive Cancer Center, Northwestern University Feinberg School of Medicine, Chicago, Illinois; ${ }^{b}$ Medical Oncology, Dana-Farber Cancer Institute, Boston, Massachusetts; 'Biostatistics and Data Coordinating Center, City of Hope National Medical Center, Duarte, California; 'Lymphoma/Myeloma, University of Texas MD Anderson Cancer Center, Houston, Texas; ${ }^{\text {eHematology and }}$ Stem Cell Transplantation, City of Hope National Medical Center, Duarte, California; fUniversity of Michigan Comprehensive Cancer Center, Ann Arbor, Michigan; 'Departments of Medicine and Immunology, Roswell

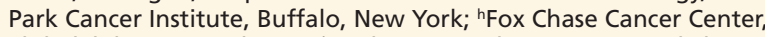
Philadelphia, Pennsylvania; 'Medicine/Lymphoma, Memorial Sloan Kettering Cancer Center, New York, New York; and iJames P. Wilmot Cancer Center, University of Rochester, Rochester, New York.

Submitted November 20, 2015; accepted for publication June 14, 2016. The authors have disclosed that they have no financial interests, arrangements, affiliations, or commercial interests with the manufacturers of any products discussed in this article or their competitors. The
NCCN non-Hodgkin's lymphoma database project was reviewed by the Institutional Review Board and received approval at each participating institution. Dr. Zhou was supported by the National Institutes of Health (NIH) Fellowship Training Grant (T32) under the award number (T32 CA 79447-15).

The content is solely the responsibility of the authors and does not necessarily represent the official views of the $\mathrm{NIH}$.

Author Contributions: Designed and performed research study, analyzed data, and wrote manuscript: Zhou and Winter. Organized research data and helped in data analysis: Rademaker, Crosby-Thompson, Vanderplas, and Niland. Designed research study, collected data, and contributed significantly to writing and editing of manuscript: Gordon, LaCasce, Zelenetz, Abel, Rodriguez, Nademanee, Kaminski, Czuczman, Millenson, and Friedberg.

Correspondence: Jane N. Winter, MD, Division of Hematology/Oncology, Northwestern University Feinberg School of Medicine, 676 North St. Clair Street, Suite 850, Chicago, IL 60611. E-mail: j-winter@northwestern.edu 
Impact of BMI and Sex on Elderly DLBCL Patients

\section{Background}

Results of prospective clinical trials for patients with previously untreated diffuse large B-cell lymphoma (DLBCL) suggest that patient sex, weight, and/or body mass index (BMI) impact clinical outcomes. Recent reports implicate differences in rituximab metabolism as the explanation for differences in outcomes for male and female patients. ${ }^{1-3}$ Analysis of pharmacokinetic data from clinical trial subjects by the German High-Grade Non-Hodgkin Lymphoma Study Group (DSHNHL) ${ }^{3,4}$ shows that rituximab clearance is relatively slow in elderly (age $>60$ years) women compared with men, leading to higher blood levels and prolonged exposure to rituximab, possibly explaining the better clinical outcomes observed in elderly women. Specifically, it has been suggested that elderly men are underdosed, based on faster rituximab clearance.

Reports on the impact of BMI on treatment outcomes are conflicting. Analysis of the US Veterans Administration (VA) database shows an association between increased BMI and improved survival in patients with DLBCL, ${ }^{5}$ whereas similar analyses of the ECOG clinical trial (E4494) for patients with DLBCL aged 60 years and older fail to show either a significant association of BMI with clinical outcomes or a sex difference related to BMI in failure-free survival. ${ }^{6}$ To further investigate these potential risk factors, we studied the effect of patient sex, BMI, and body surface area (BSA; the actual dosing parameter), and interactions among these factors, on long-term clinical outcomes in patients with DLBCL included in the prospectively collected NCCN Outcomes Database for Non-Hodgkin's Lymphoma, focusing on the elderly subset.

\section{Patients and Methods}

Patient Cohort: NCCN NHL Database for DLBCL

The formation and data structure of this multicenter NCCN Outcomes Database for NHL was previously reported. ${ }^{7}$ There are 7 participating NCCN Member Institutions, including (1) City of Hope Comprehensive Cancer Center, (2) Dana-Farber/Brigham and Women's Cancer Center, (3) Fox Chase Cancer Center, (4) The University of Texas MD Anderson Cancer Center, (5) Roswell Park Cancer Institute, (6) University of Michigan Comprehensive Cancer Center, and (7) Robert H. Lurie Comprehensive
Cancer Center of Northwestern University. Sequential patients seen at these centers older than 18 years with newly diagnosed DLBCL between June 1, 2000, and December 31, 2010, were included, with followup through December 31, 2011. Patients were required to be cancer-free for 5 years before their lymphoma diagnosis.

\section{Study Cohort}

De novo DLBCL cases diagnosed between June 2000 and December 2010 and treated with a rituximab-containing regimen as first-line therapy were included in the main study group. Patients were divided into 2 age subgroups: older than 60 years and 60 years of age or younger. The following additional patient characteristics were extracted from the database: sex, BMI (weight $[\mathrm{kg}] /$ height $[\mathrm{m}]^{2}$ ), BSA (Dubois formula) ${ }^{8}$ and international prognostic index (IPI) risk category ${ }^{9}$ based on age, Ann Arbor stage (I-IV), number of extranodal site involvement, lactate dehydrogenase level, and ECOG performance status (0-4). Initial treatment, outcomes, and corresponding event dates were queried related to disease progression or death, or, if neither, date of the last follow-up visit. Patients recorded to have moderate or severe liver disease and/or severe renal insufficiency (glomerular filtration rate $<30 \%$ ) were also identified from the database.

\section{Outcomes and Methods of Analysis}

Treatment outcomes were assessed for progression-free survival (PFS) and overall survival (OS) at 3 years for the entire study population and both age subgroups ( $\leq 60$ and $>60$ years). PFS at 3 years was the primary outcome, which was determined as the earliest of death, disease recurrence, or indication of disease progression on therapy. PFS was chosen a priori because it better reflects the treatment outcomes associated with initial therapy. OS was the secondary outcome and was defined as death from any cause. The effects of sex, BMI, and BSA on PFS and OS were first studied individually in a Cox regression model. Effects of BMI and BSA on PFS both as continuous variables and as categorical variables based on WHO criteria were examined. BSA was viewed as an index for actual rituximab dosing. The effect of sex with respect to WHO classification of BMI (low, $\leq 18.5$; normal, $>18.5-25.0$; overweight, $\left.>25.0 \mathrm{~kg} / \mathrm{m}^{2}\right)$ or BSA $(<2$, $\geq 2 \mathrm{~m}^{2}$ ) were then studied in multivariable model and 
through stratified analysis using Kaplan-Meier curves and log-rank tests. Interaction terms between sex and BMI, and sex and BSA were tested respectively in the multivariable Cox model. Across participating study centers, no dose capping was mandated for rituximab based on BSA. In a sensitivity analysis, the effect estimate of these factors on PFS and OS was adjusted for the IPI (risk categories: low, low-intermediate, highintermediate, and high) in the multivariable Cox regression model. Weight alone as a prognostic factor was also examined in the univariate and multivariate analyses, adjusting for sex and age.

\section{Results}

The study population consisted of 1,386 adult patients with DLBCL with complete clinical information at baseline, all confirmed to have received rituximab-based first-line immunochemotherapy for DLBCL (Table 1). A total of 146 patients were excluded for missing height, weight, or IPI. The median age of this study population was 58 years (men, 58 years; women, 59 years); 627 patients were older than 60 years. The median follow-up for PFS of the entire cohort was 3 years, and was 2.8 years in the elderly (age $>60$ years) subset. Most elderly men were

\begin{tabular}{|c|c|c|c|}
\hline & All & Male & Female \\
\hline Median age, y & 58 & 58 & 59 \\
\hline Male, n (\%) & $749(54 \%)$ & & \\
\hline \multicolumn{4}{|l|}{ Mean BMI, n (\%) } \\
\hline$\leq 18.5 \mathrm{~kg} / \mathrm{m}^{2}$ & $24(1.7 \%)$ & $6(0.4 \%)$ & $18(1.3 \%)$ \\
\hline$>18.5-25.0 \mathrm{~kg} / \mathrm{m}^{2}$ & $433(31.2 \%)$ & $181(13.1 \%)$ & $252(18.2 \%)$ \\
\hline$>25 \mathrm{~kg} / \mathrm{m}^{2}$ & $929(67.0 \%)$ & $562(41.1 \%)$ & $367(24.0 \%)$ \\
\hline \multicolumn{4}{|l|}{ Mean BSA, n (\%) } \\
\hline$<2 \mathrm{~m}^{2}$ & $902(65.1 \%)$ & $333(24.0 \%)$ & $569(41.1 \%)$ \\
\hline$\geq 2 \mathrm{~m}^{2}$ & $484(34.9 \%)$ & $416(30.0 \%)$ & $68(4.9 \%)$ \\
\hline \multicolumn{4}{|c|}{ International prognostic index } \\
\hline Low & $39.6 \%$ & $20.7 \%$ & $18.9 \%$ \\
\hline Low-intermediate & $26.7 \%$ & $14.9 \%$ & $11.8 \%$ \\
\hline High-intermediate & $20.9 \%$ & $11.8 \%$ & $9.2 \%$ \\
\hline High & $12.8 \%$ & $6.6 \%$ & $6.1 \%$ \\
\hline
\end{tabular}

Abbreviations: BMI, body mass index; BSA, body surface area. either overweight or large (mean BMI, 28.7 for men and 27.6 for women); only $13 \%$ had a BMI of 25 $\mathrm{kg} / \mathrm{m}^{2}$ or less and only $24 \%$ had BSA of less than $2 \mathrm{~m}^{2}$. The IPI scores across categories of BMI $(\leq 18.5$, $\left.>18.5-25.0,>25.0 \mathrm{~kg} / \mathrm{m}^{2}\right)$ and BSA $\left(<2, \geq 2 \mathrm{~m}^{2}\right) \mathrm{did}$ not differ significantly (Wilcoxon rank sum test, $P=.42$ for BMI and $P=.13$ for BSA). Patients with documented chronic moderate to severe liver and renal dysfunction were $0.7 \%(n=10)$ and $1.4 \%(n=19)$, respectively, and were of no significant difference in distribution across levels of BMI and BSA. Most patients received rituximab-based anthracycline containing immunochemotherapy, and uses were similar across BMI groups (92\% for low-weight, 97\% for normal-weight, and $98 \%$ for overweight groups, respectively; chi-square, $P=.21$ )

\section{Individual Effects of BMI, BSA, and Sex on Clinical Outcomes}

When BMI and BSA were considered as continuous variables, the effect of BMI on disease progression or death (PFS) varied across its range, with a higher risk associated with low BMI and the risk decreasing as BMI increased (Figure 1A). Similarly, risk of disease

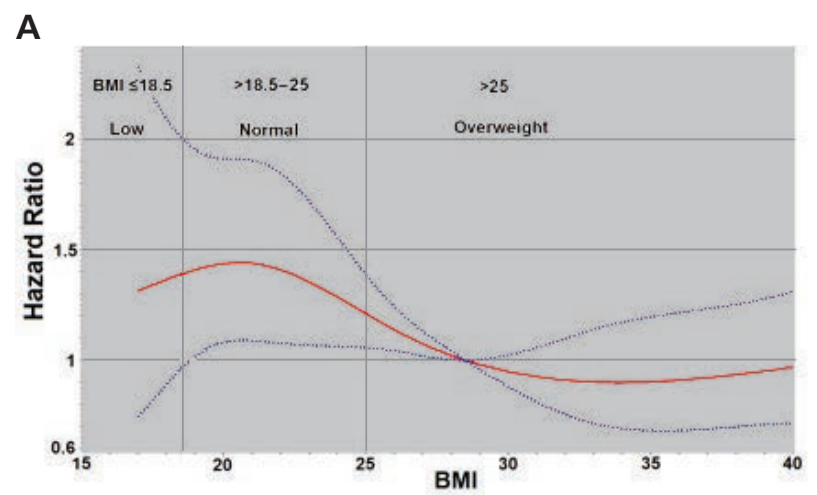

B

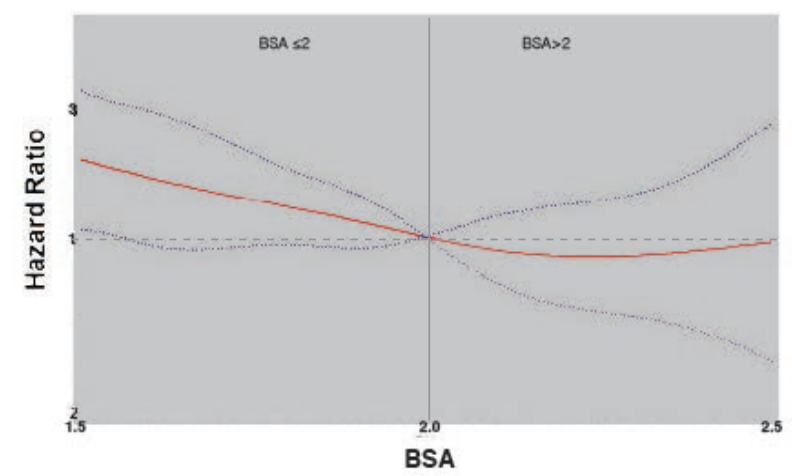

Figure 1. Change of risk on 3-year progression-free survival across range of $(A)$ body mass index (BMI) and (B) body surface area (BSA) in terms of estimated hazard ratios (HR; red) and $\mathrm{Cl}$ band (blue). 
progression or death decreased with increasing BSA (Figure 1B). Analysis of BMI categorized based on the WHO categorization (low, $\leq 18.5 \mathrm{~kg} / \mathrm{m}^{2}$; normal, $>18.5-25.0 \mathrm{~kg} / \mathrm{m}^{2}$; overweight, $>25 \mathrm{~kg} / \mathrm{m}^{2}$ ) showed that compared with normal BMI, higher BMI (>25 $\mathrm{kg} / \mathrm{m}^{2}$ ) was protective (3-year PFS: hazard ratio [HR], 0.7; $P<.01$ ) (Table 2). Importantly, higher BMI was not significantly associated with more favorable clinical features as defined by the IPI risk categories (chisquare, $P=.51$ ). Effect of sex on PFS was borderline significant when analyzed in the entire study population, among which male sex (54\%) showed worse outcomes with lower PFS (3-year HR, 1.3; P=.04). Notably, the poor risk associated with male sex was seen mostly in the elderly age group ( $>60$ years), and not in those who were younger ( $\leq 60$ years) (Table 2 and Figure 2A). Older age itself ( $>60$ vs $\leq 60$ years) was a significant predictor of poor outcomes (3-year PFS: HR, 1.4; $P<.01$; OS: HR, 2.1; $P<.001)$.

\section{Effect of Sex in Relation to the Levels of BMI and BSA and Patient Age}

Among all patients, male sex was associated with worse clinical outcomes after adjusting for age and BMI or BSA (3-year PFS: HR, 1.4 and 1.5, respectively; $P<.01$ ). (Table 3 ). Of note, the magnitude of risk associated with male sex on survival diminished with increasing BMI $\left(\leq 18.5 \mathrm{~kg} / \mathrm{m}^{2},>18.5-25.0 \mathrm{~kg} / \mathrm{m}^{2}\right.$, $\left.>25 \mathrm{~kg} / \mathrm{m}^{2}\right)$ and BSA $\left(<2 \mathrm{~m}^{2}, \geq 2 \mathrm{~m}^{2}\right)$ (Figure $2 \mathrm{~B}-\mathrm{C}$ ). Compared with men, women showed a significantly lower risk of 3-year PFS in the normal BMI category (log-rank, $P<.01$ ), whereas the sex difference in risk was no longer present in the high BMI group (logrank, $P=.29$ ). Similarly with BSA, the sex difference in survival was most apparent in the normal BSA group $\left(<2 \mathrm{~m}^{2}\right)$, in the group with BSA of $2 \mathrm{~m}^{2}$ or more. HR estimates for sex, BMI, and BSA remained largely unchanged after adjusting for IPI in the multivariable model. Normal BMI versus high BMI independently predicted poor outcomes (3-year PFS: HR, 1.5 ; $P$ <.01; OS: HR, 1.6; $P<.01)$ after adjusting for sex, as did BSA $\left(<2 \mathrm{~m}^{2}\right.$ vs $\left.\geq 2 \mathrm{~m}^{2}\right)$, which correlated with a lower risk of 3 -year PFS (HR, 1.4; P=.03) (Table 3). Weight itself, irrespective of height or BMI, was not a significant prognostic factor for PFS (3-year HR, 0.99; 95\% CI, 0.99-1.00; P=.09) or OS (3-year HR, 1.00; 95\% CI, 0.99-1.01; $P=.32$ ) after adjusting for sex and age.

Specifically, in the elderly age subgroup $(n=627)$, high BMI remained protective. The sex difference in effect on PFS attenuated with increase in levels of BMI and BSA. Notably, the poor risk associated with male

\begin{tabular}{|c|c|c|}
\hline & $\begin{array}{l}\text { 3-Year PFS, } \\
\text { HR }(95 \% \mathrm{Cl})\end{array}$ & $\begin{array}{l}\text { 3-Year OS, } \\
\text { HR (95\% CI) }\end{array}$ \\
\hline Age $>60$ y $(n=627)^{a}$ & $1.4(1.1-1.7) ; P<.01$ & $2.1(1.5-2.8) ; P<.01$ \\
\hline \multicolumn{3}{|l|}{ Sex } \\
\hline Male $(n=749,54 \%)^{b}$ & $1.3(1.0-1.6) ; P=.04$ & $1.6(1.2-2.1) ; P<.01$ \\
\hline$\leq 60(n=424)^{c}$ & $1.2(0.9-1.7) ; P=.27$ & $1.9(1.1-3.2) ; P=.02$ \\
\hline$>60(n=325)^{d}$ & $1.4(1.0-2.0) ; P=.04$ & $1.5(1.0-2.2) ; P=.04$ \\
\hline \multicolumn{3}{|l|}{ BMI } \\
\hline$>18.5-25.0 \mathrm{~kg} / \mathrm{m}^{2}(\mathrm{n}=433)^{\mathrm{e}}$ & $1.4(1.1-1.8) ; P=.01$ & $1.5(1.1-2.0) ; P=.01$ \\
\hline$>25 \mathrm{~kg} / \mathrm{m}^{2}(\mathrm{n}=929)^{f}$ & $0.7(0.6-0.9) ; P<.01$ & $0.7(0.5-0.9) ; P=.02$ \\
\hline BSA $<2 m^{2}(n=902)^{9}$ & $1.1(0.9-1.4) ; P=.35$ & $1.1(0.8-1.5) ; P=.70$ \\
\hline
\end{tabular}

\footnotetext{
Abbreviations: BMI, body mass index; BSA, body surface area; DLBCL, diffuse large B-cell lymphoma; HR, hazard ratio; OS, overall survival; PFS, progression-free survival.

aCompared with age $\leq 60$ y $(n=759)$.

bompared with female $(\mathrm{n}=637)$.

'Compared with female $>60$ y $(n=302)$.

${ }^{\mathrm{d} C o m p a r e d}$ with female $\leq 60$ y $(n=335)$.

eCompared with high BMI $(>25 \mathrm{~kg} / \mathrm{m} 2)$.

${ }^{\dagger}$ Compared with normal BMI $(>18.5-25.0 \mathrm{~kg} / \mathrm{m} 2)$.

${ }^{9}$ Compared with BSA $\geq 2 \mathrm{~m} 2(\mathrm{n}=484)$.
} 
A
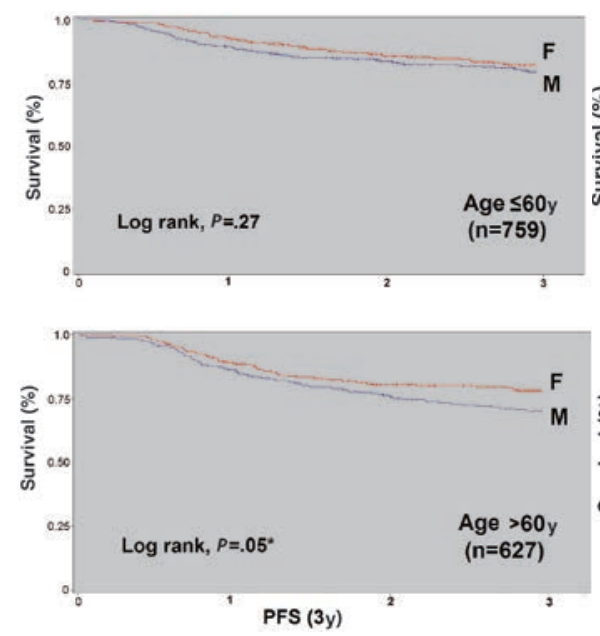

B
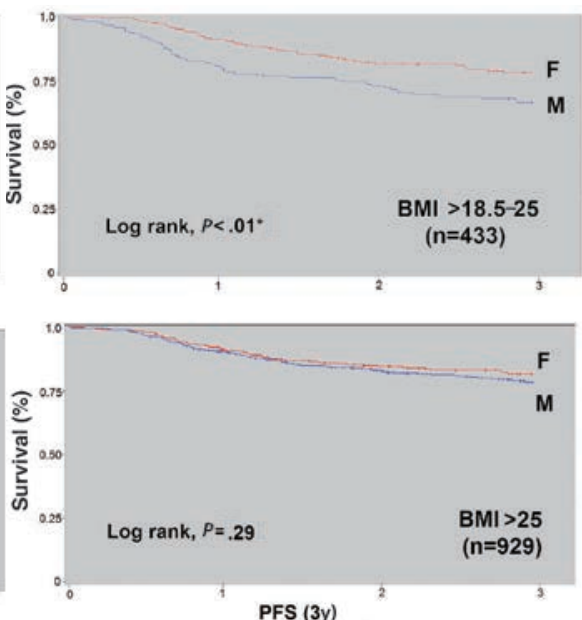

C
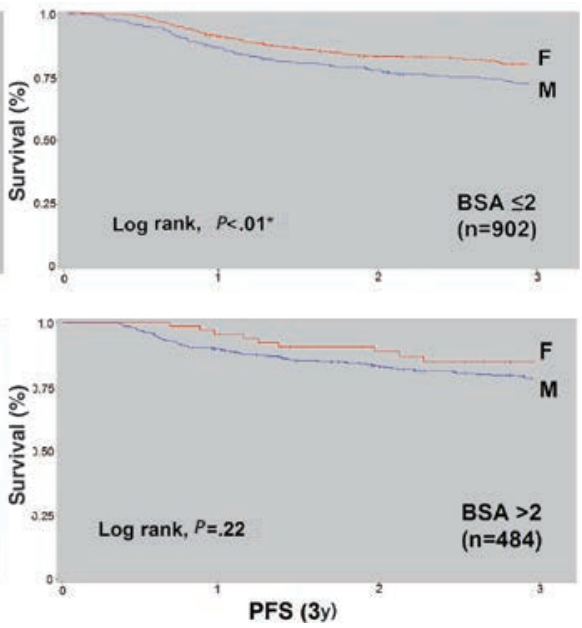

Figure 2. Kaplan-Meier curves showing effect of sex on 3-year progression-free survival (PFS) stratified by the (A) age groups, (B) levels of body mass index (BMI), and (C) body surface area (BSA) in all patients. ${ }^{*} P$ is significant $(\mathrm{ie},<.05)$.

sex was most pronounced in patients older than 60 years (3-year PFS: HR, 1.5; 95\% CI, 1.1-2.1; P=.02), but not the younger cohort (3-year PFS: HR, 1.3; 95\% CI, 0.9-1.9; $P=.12$ ) (Table 3 and Figure 3A, B). An interaction between sex and BMI was demonstrated in the Cox regression analysis $(P=.02)$ in the elderly age group, whereas the interaction term for sex and BSA did not reach significance $(P=.37)$. Weight did not appear to be a significant prognostic factor in the elderly subgroup either (3-year PFS: HR, 1.00; 95\% CI, 0.99-1.01; P=.47)

\section{Discussion}

The addition of rituximab to front-line anthracycline-containing regimens for patients with DLBCL has been shown to improve outcomes in both men and women, young and old. Several analyses have shown greater benefit in women, especially among the elderly, attributed to slower clearance of rituximab, resulting in higher and more prolonged rituximab levels. ${ }^{1,3}$ These findings raise the issue of potential rituximab underdosing and suboptimal clinical outcomes in elderly men. In this analysis of clinical outcomes of nearly 1,400 patients with newly diagnosed DLBCL enrolled in the NCCN Outcomes Database for NHL, we have confirmed previous reports of an age-related disadvantage to male sex. Furthermore, we found that this effect on patient outcomes is abrogated by higher levels of BMI and BSA in most North American patients with DLBCL.
Our results, derived from real-world clinical experience at $7 \mathrm{NCCN}$ Member Institutions, confirm and extend those reported by others that were based exclusively on patients enrolled on clinical trials. . $^{1,6,10}$ We report that in the rituximab era, male sex is associated with a higher risk of disease progression and death than female sex, and that the difference in PFS results primarily from the elderly patient subgroup (>60 years). The difference between older women and men is as yet unexplained and may reflect the comparatively better survival of women versus men in the general population. An alternative explanation suggested by detailed pharmacokinetic studies of elderly clinical trial subjects ${ }^{1,3}$ receiving rituximab-containing chemotherapy is that a more pronounced agedependent decline in rituximab clearance in women compared with men underlies the comparatively better outcomes observed in elderly women.

In terms of the role of patient body habitus, previous studies have investigated the impact of weight and BMI on clinical outcome with mixed results. Analysis of the RICOVER-60 trial suggests that the addition of rituximab to conventional therapy has differential effects related to body weight. ${ }^{3}$ Patients in the lowest weight quartile experienced significant improvement in PFS with the addition of rituximab, whereas the top weight quartile had only a minor improvement in outcomes. In our analysis of the NCCN Outcomes Database, we used BMI and BSA to better reflect body habitus. We show that high BMI $\left(>25 \mathrm{~kg} / \mathrm{m}^{2}\right)$ favorably influences treatment out- 


\begin{tabular}{|c|c|c|}
\hline & $\begin{array}{l}\text { 3-Year PFS, } \\
\text { HR }(95 \% \mathrm{Cl})\end{array}$ & $\begin{array}{l}\text { 3-Year OS, } \\
\text { HR (95\% Cl) }\end{array}$ \\
\hline \multicolumn{3}{|l|}{ All $(n=1,386)$} \\
\hline Male $(n=749,54 \%)$ & $1.4(1.1-1.8) ; P<.01$ & $1.7(1.3-2.4) ; P<.01$ \\
\hline \multicolumn{3}{|l|}{ BMI } \\
\hline $\begin{array}{l}>18.5-25.0 \mathrm{~kg} / \mathrm{m}^{2} \\
(\mathrm{n}=433)^{\mathrm{a}}\end{array}$ & $1.5(1.2-1.9) ; P<.01$ & $1.6(1.2-2.2) ; P<.01$ \\
\hline Age $>60$ y $(n=627)$ & $1.4(1.1-1.8) ; P<.01$ & $2.2(1.6-3.0) ; P<.01$ \\
\hline Male $(n=749,54 \%)$ & $1.5(1.1-1.9) ; P<.01$ & $1.8(1.3-2.6) ; P<.01$ \\
\hline BSA $<2 m^{2}(n=902)^{b}$ & $1.4(1.0-1.8) ; P<.03$ & $1.4(0.9-2.0) ; P<.06$ \\
\hline Age >60 y $(n=627)$ & $1.4(1.9-1.7) ; P<.01$ & $2.1(1.5-2.8) ; P<.01$ \\
\hline \multicolumn{3}{|l|}{ Age $\leq 60$ y $(n=759)$} \\
\hline Male $(n=424,56 \%)$ & $1.3(0.9-1.9) ; P<.12$ & $2.2(1.3-3.7) ; P<.01$ \\
\hline \multicolumn{3}{|l|}{ BMI } \\
\hline $\begin{array}{l}>18.5-25.0 \mathrm{~kg} / \mathrm{m}^{2} \\
(\mathrm{n}=249)^{\mathrm{a}}\end{array}$ & $1.5(1.0-2.1) ; P<.04$ & $1.7(1.0-2.8) ; P<.04$ \\
\hline Male & $1.4(0.9-2.0) ; P<.12$ & $2.3(1.3-4.1) ; P<.01$ \\
\hline BSA $<2 m^{2}(n=472)$ & $1.3(0.9-1.9) ; P<.24$ & $1.6(0.9-2.8) ; P<.08$ \\
\hline \multicolumn{3}{|l|}{ Age $>60$ y $(n=627)$} \\
\hline Male $(n=325,52 \%)$ & $1.5(1.1-2.1) ; P=.02$ & $1.6(1.1-2.4) ; P<.01$ \\
\hline \multicolumn{3}{|l|}{ BMI } \\
\hline $\begin{array}{l}>18.5-25.0 \mathrm{~kg} / \mathrm{m}^{2} \\
(\mathrm{n}=184)^{\mathrm{a}}\end{array}$ & $1.6(1.1-2.2) ; P=.01$ & $1.8(1.2-2.6) ; P<.01$ \\
\hline Male & $1.6(1.1-2.3) ; P<.01$ & $1.6(1.0-2.5) ; P<.03$ \\
\hline BSA $<2 m^{2}(n=430)$ & $1.4(0.9-2.0) ; P<.12$ & $1.2(0.7-1.8) ; P<.50$ \\
\hline
\end{tabular}

For the entire group, "Age" was included as a variable in the multivariable model reported together with "Male and BMI" and "Male and BSA", respectively. Multivariable model estimates for "Male and BMI", and "Male and BSA" were then reported in 2 stratified age groups ( $\leq 60$ and $>60$ years).

Abbreviations: BMI, body mass index; BSA, body surface area; DLBCL, diffuse large B-cell lymphoma; HR, hazard ratio; OS, overall survival; PFS, progression-free survival.

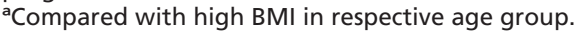

${ }^{b}$ Compared with BSA $\geq 2 \mathrm{~m} 2$ in respective age group.

comes in patients treated with rituximab-containing therapy, as does high BSA $\left(\geq 2 \mathrm{~m}^{2}\right)$, the actual dosing parameter for rituximab in practice. The results related to BMI are consistent with those reported by Carson et $\mathrm{al}^{5}$ in their analysis of the US VA database. In contrast, results derived from the ECOG E4494 trial of elderly patients with DLBCL suggest that BMI is not significantly associated with differences in treatment outcomes, nor were there differences in outcomes according to sex related to BMI, ${ }^{6}$ at least among patients eligible and enrolled on this clinical trial.
Our analysis suggests that high BMI is protective among elderly patients with DLBCL treated with rituximab-containing chemotherapy. In particular, high BMI/BSA in this North American elderly DLBCL population compensates for the negative impact of male sex. Whether the slightly higher total dosing of larger patients underlies this observation is unclear. Higher body weight has been associated with faster rituximab clearance, ${ }^{3}$ and would therefore not be expected to improve outcomes for men through its effect on rituximab pharmacokinetics. Our analysis of real-world treatment data did not suggest that patient 
A
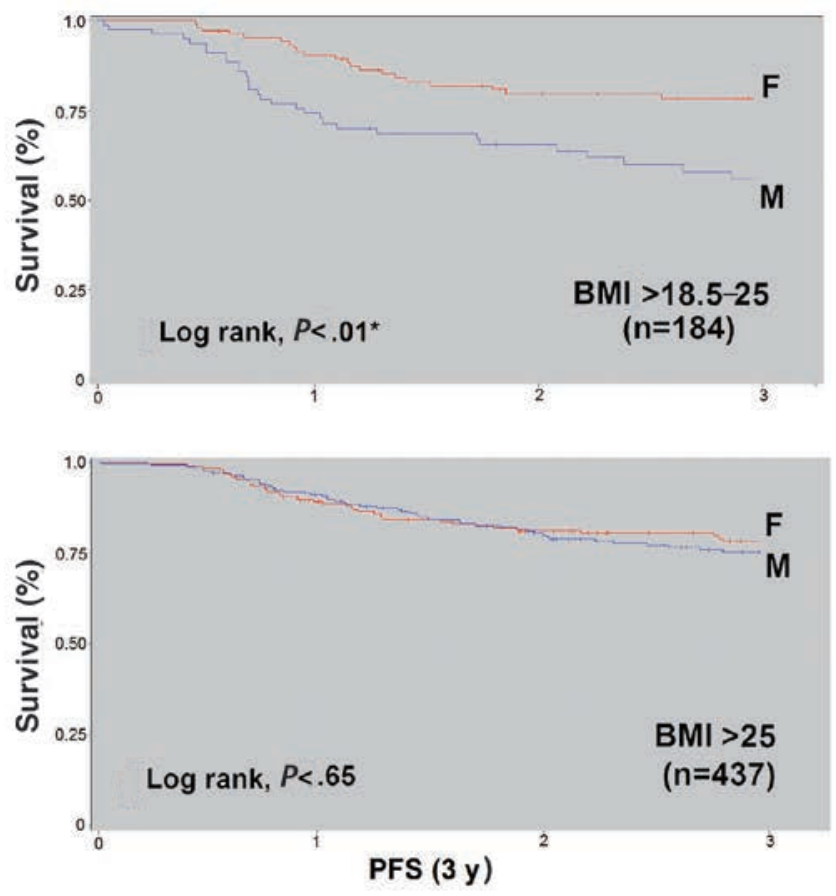

B
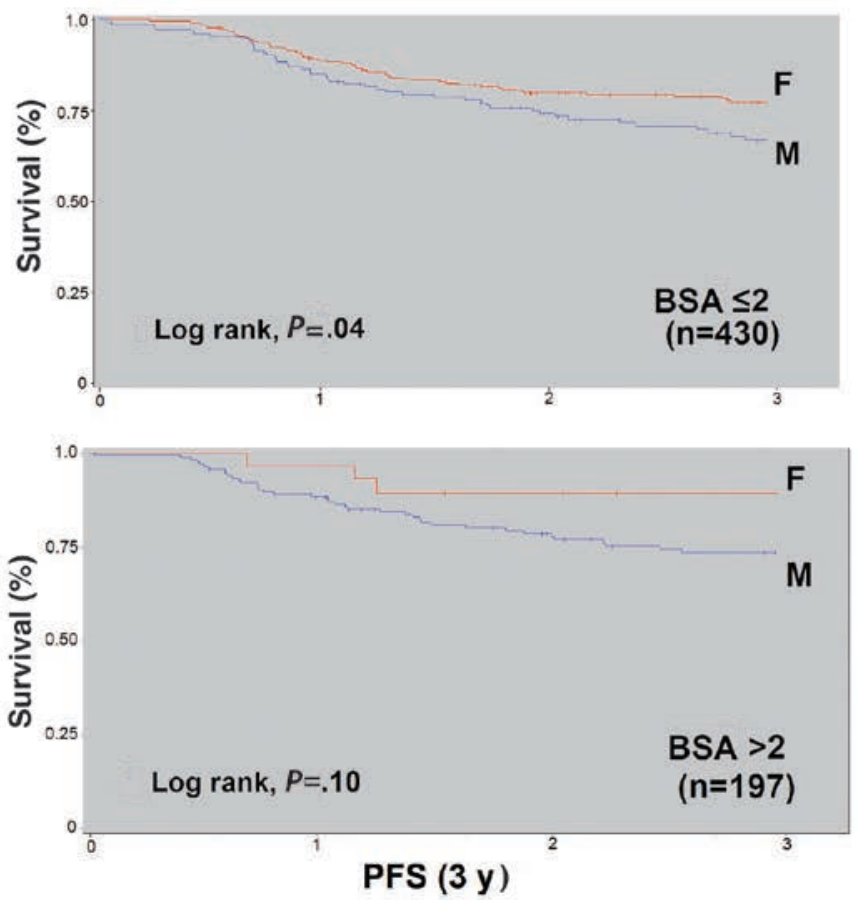

Figure 3. Kaplan-Meier curves showing effect of sex on 3-year progression-free survival (PFS) stratified by the levels of (A) body mass index (BMI) and (B) body surface area (BSA) in the elderly group (aged $>60$ years).

weight was a significant prognostic factor for PFS or OS after adjusting for sex and age. Further, the benefit associated with high BMI or BSA in our study was unlikely to be confounded by more-favorable prognostic features defined by IPI. Although it is possible that very low BMI $\left(\leq 18.5 \mathrm{~kg} / \mathrm{m}^{2}\right)$ may be a surrogate for those who were very sick and frail thereby confounding the analysis due to their poor prognosis, high BMI $\left(>25 \mathrm{~kg} / \mathrm{m}^{2}\right)$ remained protective compared with the normal BMI group (>18.5-25.0 kg/m²) when these patients were excluded. There was also no indication that there were fewer patients who received anthracycline-containing therapy in the low or normal BMI groups compared with those in the overweight group. Our results suggest that the apparent greater benefit of rituximab therapy for women versus men may not be exclusively related to renal clearance mediated by sex and patient body habitus as suggested by others, but that BMI itself may have an independent biological role in treatment outcomes after controlling for sex and age. ${ }^{11}$ The favorable impact of high BMI has been previously reported in patients with lymphoma, although these studies were usually small and some predated the rituximab era and did not examine the impact of sex, age, and weight as well as BSA in a single comprehensive analysis. ${ }^{10,12-15}$ As with any observational study, our findings may be partially influenced by the survival trend associated with sex and age in the general population. Consequently, we used PFS as the primary end point to better reflect treatment outcome after initial therapy. A median followup of 3 years for PFS is a relatively short period that is less likely subject to significant impact from survival trends in the general population. The most imminent risk for failure in this disease population is the DLBCL itself and treatment-related toxicity.

\section{Conclusions}

This analysis of a large unselected North American DLBCL patient cohort treated with rituximab-based chemoimmunotherapy confirms an age-dependent disadvantage to male sex in treatment outcomes. This effect diminishes with higher levels of BMI and BSA, and impacts patients mainly of normal or low $\mathrm{BMI} / \mathrm{BSA}$, the minority of patients with DLBCL in North America. These findings do not exclude the possibility that higher doses of rituximab or different scheduling could benefit subsets of patients, as suggested by preliminary results from the random- 
ized phase II SEXIE-R-CHOP- $14^{16}$ and analysis of the SMARTE-R-CHOP-14 trial. ${ }^{17}$ Our findings support continued efforts to optimize rituximab dosing, especially in elderly men with a BMI of $25 \mathrm{~kg} / \mathrm{m}^{2}$ or less or BSA of $2 \mathrm{~m}^{2}$ or less. Future prospective trials should factor body habitus and sex into the study design and analysis.

\section{Acknowledgments}

The authors wish to acknowledge the patients and staff of the NCCN Member Institutions participating in the lymphoma database for their contributions.

\section{References}

1. Pfreundschuh M, Muller C, Zeynalova S, et al. Suboptimal dosing of rituximab in male and female patients with DLBCL. Blood 2014;123:640 646.

2. Habermann TM, Hong F, Morrison VA, et al. Differences in outcomes in males and females with diffuse large B-Cell lymphoma with induction rituximab and follicular lymphoma treated with maintenance rituximab. Blood 2012;21:3705.

3. Muller C, Murawski N, Wiesen MH, et al. The role of sex and weight on rituximab clearance and serum elimination half-life in elderly patients with DLBCL. Blood 2012;119:3276-3284.

4. Habermann TM. Is rituximab one for all ages and each sex? Blood 2014;123:602-603.

5. Carson KR, Bartlett NL, McDonald JR, et al. Increased body mass index is associated with improved survival in United States veterans with diffuse large B-cell lymphoma. J Clin Oncol 2012;30:3217-3222.
6. Hong F, Habermann TM, Gordon LI, et al. The role of body mass index in survival outcome for lymphoma patients: US intergroup experience. Ann Oncol 2014;25:669-674.

7. Kho ME, Lepisto EM, Niland JC, et al. Reliability of staging, prognosis, and comorbidity data collection in the National Comprehensive Cancer Network (NCCN) non-Hodgkin lymphoma (NHL) multicenter outcomes database. Cancer 2008;113:3209-3212.

8. Verbraecken J, Van de Heyning P, De Backer W, et al. Body surface area in normal-weight, overweight, and obese adults. A comparison study. Metabolism 2006;55:515-524.

9. A predictive model for aggressive non-Hodgkin's lymphoma. The International Non-Hodgkin's Lymphoma Prognostic Factors Project. N Engl J Med 1993;329:987-994.

10. Sarkozy C, Mounier N, Delmer A, et al. Impact of BMI and gender on outcomes in DLBCL patients treated with R-CHOP: a pooled study from the LYSA. Lymphoma 2014: doi:10.1155/2014/205215. Available at: http://www.hindawi.com/archive/2014/205215/cta/. Accessed August 29, 2016.

11. Dahl AK, Fauth EB, Ernsth-Bravell M, et al. Body mass index, change in body mass index, and survival in old and very old persons. J Am Geriatr Soc 2013;61:512-518.

12. Jones JA, Fayad LE, Elting LS, et al. Body mass index and outcomes in patients receiving chemotherapy for intermediate-grade B-cell nonHodgkin lymphoma. Leuk Lymphoma 2010;51:1649-1657.

13. Han X, Stevens J, Bradshaw PT. Body mass index, weight change, and survival in non-Hodgkin lymphoma patients in Connecticut women. Nutr Cancer 2013;65:43-50.

14. Weiss $L$, Melchardt $T$, Habringer $S$, et al. Increased body mass index is associated with improved overall survival in diffuse large B-cell lymphoma. Ann Oncol 2014;25:171-176.

15. Park S, Han B, Cho JW, et al. Effect of nutritional status on survival outcome of diffuse large B-cell lymphoma patients treated with rituximabCHOP. Nutr Cancer 2014;66:225-233.

16. Pfreundschuh $M$, Held $G$, Zeynalova S, et al. Increased rituximab (R) doses and effect on risk of elderly male patients with aggressive CD20+ B-cell lymphomas: results from the SEXIE-R-CHOP-14 trial of the DSHNHL [abstract]. J Clin Oncol 2014;32(Suppl):Abstract 8501.

17. Pfreundschuh M, Poeschel V, Zeynalova S, et al. Optimization of rituximab for the treatment of diffuse large B-cell lymphoma (II): extended rituximab exposure time in the SMARTE-R-CHOP-14 trial of the German High-Grade Non-Hodgkin Lymphoma Study Group. J Clin Oncol 2014;32:4127-4133. 